\title{
Software musical en la enseñanza-aprendizaje de los estudiantes de Música de la Universidad Nacional del Altiplano Puno
}

\section{Musical software in the teaching-learning of Music students of the National University of the Altiplano Puno}

Renzo Favianni Valdivia Terrazas a

0000-0001-8222-3264

\author{
Wilber Cesar Calsina Ponce ${ }^{b}$ \\ 0000-0001-9094-7684
}

Benjamín Velazco Reyes ${ }^{\mathrm{c}}$

0000-0003-2780-786X

Universidad Nacional del Altiplano, Puno, Perú.

${ }^{a}$ rfvaldivia@unap.edu.pe

${ }^{\mathrm{b}}$ wcalsina@unap.edu.pe

cbvelazco@unap.edu.pe
Recibido: $10 / 01 / 2021$

Aceptado: $28 / 02 / 2021$

Pub1icado: 17/03/2021

\section{Resumen}

Los softwares musicales han revolucionado la manera de cómo los músicos en la actualidad desarrollan la práctica musical en cuanto a la composición, arreglos musicales y orquestación, es por ello que el presente estudio se planteó como objetivo; determinar la influencia de los Software musicales en el proceso de enseñanza-aprendizaje de los estudiantes de música de la Universidad Nacional del Altiplano Puno. El enfoque fue cuantitativo, siendo el diseño cuasi experimental y se aplicó instrumentos como el pre test, y post test, para la recolección y análisis de los datos, teniendo como resultado que los estudiantes tienen nuevas aptitudes en el proceso de la composición, arreglos musicales y orquestación, utilizando los Softwares Sibelius y Finale, en comparación de las técnicas tradicionales; Se concluye que existe influencia significativa del uso de los Softwares Sibelius y Finale en el proceso de enseñanza-aprendizaje de los estudiantes; ya que se observó que hubo mejora en el desarrollo de las capacidades en la composición, arreglos musicales y orquestación.

Palabras clave: Arreglos musicales; composición; enseñanza-aprendizaje; orquestación; softwares musicales.

\begin{abstract}
Music software have revolutionized the way musicians nowadays develop their musical practice in terms of composition, musical arrangements and orchestration, that is why in the present research the objective was set; to determine the influence of music software in the teaching-learning process of music students at the Nacional University of the Altiplano in Puno. The approach was quantitative, being the design quasi-experimental and applied instruments such as pre-test, and post-test, for the collection and analysis of data, resulting in students have new skills in the process of composition, musical arrangements and orchestration, using the Sibelius and Finale software, compared with traditional techniques; It is concluded that there is a significant influence of the use of the Sibelius and Finale software in the teaching-learning process of the students; since it was observed that there was improvement in the development of the abilities in composition, musical arrangements and orchestration.
\end{abstract}

Keywords: composition; musical arrangements; musical software; orchestration; teaching-learning. 


\section{Introducción}

La música es una de las expresiones creativas más íntimas del ser, ya que forma parte del quehacer cotidiano de cualquier grupo humano tanto por su goce estético como por su carácter funcional y social (Dammers, 2012). Además, la música nos identifica como seres, como grupos y como cultura, tanto por las raíces identitarias como por la locación geográfica y épocas históricas (Bannerman \& O'Leary, 2020). Es un aspecto de la humanidad innegable e irremplazable que nos determina como tal (Rolando y Alvarado, 2013).

En la música, así como en otras actividades académicas la tecnología viene dinamizando el proceso de enseñanza aprendizaje, ya que, hasta una década atrás en las capacidades de composición, arreglos y orquestación, se tenía que acudir a un grupo determinado de músicos para que puedan interpretar las obras y dar la validación de ellas; proceso que se agilizó y revolucionó estas capacidades, con la aparición de softwares para edición y reproducción y otros de partituras - Sibelius y Finale (García y Pelayo, 1995).

Así mismo, los softwares musicales han proporcionado nuevos recursos, como el acceso a la reproducción de un amplio repertorio, el trabajo de los intérpretes, el acceso a las puntuaciones, etc. En los últimos años, nuevas herramientas se utilizan para crear, editar y reproducir partituras en línea (Hormigos, 2004). Un compositor de música por medio de un ordenador usando un software puede componer sin preocuparse menos por las tediosas dificultades que implica la importación de datos en un programa de notación gráfica. También se puede generar información como marcas, dinámicas y articulaciones las mismas que aparecen en la partitura (Rodríguez, 2012).

Los formatos más utilizados para el intercambio de información musical en la actualidad están limitados porque representan la música como flujos de eventos planos y rígidos o como señales de audio sin procesar sin ninguna información estructural sobre el contenido. (Tsang \& McCracken, 2004). Dichos archivos solo se pueden escucharse de manera lineal, reutilizar y manipular de la manera que determine una aplicación de destino, como una estación de trabajo de audio digital. (Chan, Jones, Scanlon, \& Joiner, 2006).
Tejada \& Pérez-Gil (2016) el sotfware musical EMOLab, ayuda al aprendizaje de la música, en especial en los procesos perceptivos de discriminación de ritmo y melodía, en los procesos de asociación simbólica del sistema de notacional musical occidental y en los procesos de memoria musical debido a dos factores: a) la integración de modos de presentación de información auditiva y visual; b) un diseño fundamentado en principios básicos de educación musical.

Otro aspecto importante en el aprendizaje de la música es el internet que ha entrado en las aulas de educación musical para quedarse. La respuesta inmediata a encontrar información en Internet, es querer ver un video o intentar compartir esa información es un paso adelante que no tiene posibilidad de retroceder. A su vez, existen herramientas tecnológicas como ordenadores, tabletas digitales $y$ teléfonos inteligentes, programas informáticos y aplicaciones hacen que la enseñanza y el aprendizaje de la música sean mucho más significativo, motivador y efectivo (Herrera, 2016).

Los beneficios llegarán a los estudiantes y por ende a la educación musical (Smith, 2009). Se comprueba la importancia de la integración tecnológica al proceso de aprendizaje, cuya ayuda educativa tiene un gran potencial por las múltiples posibilidades y ventajas que ofrece (Barry, 1992). Todo ello debe servir como un elemento que se pueda compartir con los demás en el aula, no obstante, se debe realizar una reflexión y análisis crítico previo de todo lo que se ha de hacer (Haning, 2015). Sentirse atraído o incluso abrumado por todo este nuevo universo puede ser fácil, pero todo no es válido. Debe verificarse de antemano y aprender lo que quiere traer al aula (Tejada, 2009).

La aplicación de los softwares musicales Sibelius y Finale se desarrolló con el fin de mejorar en la enseñanza-aprendizaje de los estudiantes de Música de la Universidad Nacional del Altiplano Puno.

Por lo mencionado anteriormente el objetivo que encaminó la investigación se determina en la influencia de los softwares musicales en el proceso de enseñanza-aprendizaje de los estudiantes de música de la Universidad Nacional del Altiplano Puno, para ello se considera indicadores como; teoría musical, entrenamiento auditivo, reconocimiento visual y auditivo, los cuales son 
pilares en la formación de los estudiantes de música.

\section{Marco teórico}

\section{La música}

La música siempre ha acompañado al ser humano, desde épocas antiguas expresando emociones, sentimientos y hasta rituales, convirtiéndose en una manera de concebir el mundo. La dimensión más significativa de la música es su funcionalidad dentro de un contexto social determinado, esto lo realiza integrando a un escenario cultural que genera y determina el papel comunicativo que posee la música en la vida del individuo. Aunque hay muchas definiciones para la música, no hay una declaración precisa sobre lo que significa el término. Imagina dos de los más tradicionales y simples para Garcia \& Pelayo, (1995): El arte de hacer que los sonidos sean agradables al oído y el arte de conectar racional y lógicamente una combinación coherente de sonidos y quietud utilizando los principios básicos de melodía, armonía y ritmo.

El primero de ellos define el fenómeno musical como algo agradable para el oído, lo que implica que las manifestaciones que combinan incómodamente los sonidos no se pueden llamar música. Mientras que el segundo nos menciona si tal combinación debiese ser agradable o no, sino que simplemente enfatiza que una manifestación de sonido debe entenderse como música, debe usarse ciertos elementos, especialmente melodía, armonía y ritmo. Ambas definiciones son exclusivas y anacrónicas en relación con la realidad musical actual (Campos y Restrepo, 2002).

\section{Componentes principales de la música}

La forma y estructura es la organización de ideas de toda composición musical, este conjunto de ideas es que puede determinar la llamada forma musical por ende se vale de elementos importantes para llegar a este fin tal como es el sonido como la materia prima del arte musical, a partir de aquí, la música se sirve de cuatro elementos principales que ayudan al compositor a hacer música (Zimmermann, 2005). Estos elementos son: ritmo, melodía, contrapunto y armonía:

a) Ritmo: es un principio ordenado de los sonidos. No debemos confundirlo con el compás. (Ariza
\& Cuthbert, 2010). El ritmo no ha sido inventado por el hombre, ya que es un elemento que existe por sí mismo y está presente en la naturaleza, en la alternativa de las estaciones del año, en las mareas, en el fluir de la sangre, en la respiración el ritmo musical es la combinación de movimientos binarios y ternarios (Agar, 2003). b) Melodía: es una sucesión de sonidos con sentido musical, ordenados por el ritmo. Melodía y ritmo son como las dos caras de una misma moneda, siendo ambos imprescindibles para que exista la música (Chen, 2012). c) Contrapunto: sistema de composición musical que se basa en utilizar varias (dos o más) líneas melódicas independientes entre sí, simultáneas, que suenan a la vez, y donde todos los sonidos tienen la misma importancia (Naughtin, 2017). d) Armonía: sistema de composición que se establece es el s. XVII. Utiliza varios sonidos que suenan a la vez, pero siendo uno el principal y el resto subordinándosele (Irene, 2016).

\section{Generalidades sobre las habilidades musicales}

Las habilidades musicales se dividen en dos grupos: habilidades auditivas (el tubo elemental melódico, el oído melódico y armónico de la música, el oído absoluto y el oído interno) y habilidades técnicas (Borbonet, 1996). El oído melódico es para sostener y reproducir una melodía cantada. El oído armónico juega un papel en los acordes y en todo tipo de música polifónica.

La capacidad de reconocer y distinguir entre los diferentes estilos musicales es esencial para desarrollar habilidades auditivas y musicales. Sin embargo, esta tarea puede resultar difícil para los estudiantes de música, en particular para los no expertos (Huovinen \& Rautanen, 2020).

\section{Software musical}

La mayor presencia de la tecnología en las aulas de educación musical ha coincidido en cierta medida con la mayor presencia de la música popular en los espacios musicales (Wise, Greenwood, \& Davis, 2019) "El uso del software educativo tiene mucha importancia porque propicia el desarrollo del aprendizaje, es decir el uso de las tareas, actividades estructuradas y guiadas que proporcionan a los alumnos una tarea bien definida, así como los recursos que les permiten realizarlas" (Canales, 2006, p. 9). Para el caso específico de los softwares musicales, los estudiantes deben comprender el espaciado compositivo para estudiar 
cómodamente, especialmente con canciones recién arregladas y una correcta visualización de la connotación musical (Julia et al., 2019).

El uso de los softwares musicales no solo se da en las instituciones académicas, más por el contrario también ingresa a los espacios de los conjuntos de música popular, a pesar de la falta de acceso a la tecnología y/o hardware defectuoso (Powell, 2019).

\section{Software Sibelius}

Es un editor de partituras, es decir un programa (informático) completo para escribir, ejecutar, imprimir y publicar partituras de música. Fue creado por la empresa Sibelius Software, actualmente comprado por Avid Technology y está diseñado para toda clase de músicos, desde estudiantes y profesores hasta compositores profesionales (Siamancas, 2017).

El proceso de creación de canciones con Sibelius es relativamente rápido, en un mes, se puede crear nueve canciones que eran bastante variadas tanto en melodía como en ritmo (Anders \& Inden, 2019). Las melodías de las canciones creadas son grabadas directamente en la computadora, y pueden ser evaluadas y modificadas directamente según el deseo del creador (Julia et al., 2018). El modelado de teorías musicales "desde cero" es una tarea compleja, se han propuesto sistemas genéricos de programación de restricciones musicales que predefinen los componentes básicos necesarios para modelar una variedad de teorías musicales (Urgencias, 2016).

\section{Software Finale}

Es un programa completo para escribir, ejecutar, imprimir y publicar partituras de música. Fue creado por la empresa MakeMusic. Está diseñado para toda clase de músicos, desde estudiantes $\mathrm{y}$ profesores hasta compositores profesionales. Es el programa más importante de una serie de programas de edición de partituras para Microsoft Windows y Mac OS X. Finale es el programa de notación musical más popular del mercado internacional (Siamancas, 2017).

Los instrumentos virtuales en la actualidad tienen una amplia variedad de usos y varían desde digitalizar muestras de audio de instrumentos reales hasta recrear nuevos instrumentos virtuales que no existen (Psenicka, 2016). Los instrumentos virtuales ayudan a los compositores de música a componerla de manera más fácil y rápida, también brindan a los compositores nuevas ideas y conceptos para la composición musical tradicional (Yun \& Cha, 2013).

La utilización de controladores de música ha facilitado potencialmente la introducción de instrumentos musicales tradicionales en la era digital a los estudiantes. (Julia, Iswara, \& Supriyadi, 2019) La tarea de producir música impresa precisa, clara y legible para músicos de orquesta se ha vuelto mucho más rápida y sencilla con la tecnología digital (Naughtin M. , 2017).

Existen procesadores como el Saxon 8.4 XSL que se utiliza para crear la salida SVG final utilizando la hoja de estilo backend. Score SVG se configura como una aplicación basada en web utilizando la nueva tecnología Java Web Start, que permite que una aplicación Java lea y escriba archivos en un sistema cliente (Bays \& Zhu, 2015).

\section{Materiales y métodos}

La investigación responde al diseño cuasi experimental considerando los grupos de pre y post Test. Según el propósito es aplicado; ya que las investigaciones aplicadas aportan con técnicas o métodos para resolver problemas concretos, como es el caso del presente estudio (Hernández, Fernandez, \& Baptista, 2014).

\section{Técnicas e instrumentos}

La técnica de investigación utilizada fue la encuesta para obtener información de los grupos del pre y post Test; así mismo se utilizó la técnica de análisis documental de libros, tesis, artículos pertinentes para la investigación. El instrumento de investigación fue el cuestionario para lo cual previamente se utilizó en clases el software, el instrumento aplicado se centró en las opiniones de los estudiantes del Programa de Música de la Universidad Nacional del Altiplano de Puno antes y después de la intervención con el Softwares Sibelius y Finale, se consideró la valoración de datos por ítems cerrados.

\section{Población}

La población está constituida por 275 estudiantes del Programa de Música de la Universidad 
Nacional del Altiplano de Puno, tomando el siguiente criterio de inclusión y exclusión.

a. Inclusión.

- Estudiantes del Programa de Música

- Estudiantes que acepten participar en el programa

\section{b. Exclusión}

- Estudiantes no deseen formar parte de la investigación.

- Estudiantes que poseen oído absoluto

\section{Muestra}

Para la selección de la muestra se utilizó el tipo de muestreo no probabilístico porque la población es seleccionada bajo ciertos criterios de conveniencia, solo un numero preestablecido de personas por 15 alumnos del Programa de Música de la Universidad Nacional del Altiplano de Puno.

\section{Recolección de datos}

Según Arias (2004), “en este punto se describen las distintas operaciones a las que serán sometidos los datos que se obtengan" (p. 99). En virtud de ello se tomó en cuenta el análisis; que se realizó para caracterizar las situaciones y expresar la calidad de los hallazgos de la investigación, considerando las respuestas que no puedan ser expresadas cuantitativamente y el análisis interpretativo; este se efectuó en función de las variables para así evaluar los resultados en forma parcial, que facilitó la comprensión global de la información, para emitir juicios críticos y conclusiones.

La aplicación de la encuesta se realizó en varias sesiones, conforme a la disposición de tiempo de los sujetos de investigación. Se cuidó siempre que el instrumento se aplique en un ambiente donde no se detecte mucha tensión, con el fin de evitar una variación de sus respuestas actitudinales. Analizando las variables independiente Software musical en la variable dependiente enseñanza-aprendizaje.

Una vez recogidos los datos de evaluación de los sujetos de investigación, se procedió a analizar e interpretar los datos en una matriz de sistematización, en una hoja de cálculo.

\section{Resultados y discusión}

Resultados de aplicación de pre test, sobre las capacidades musicales de los estudiantes del programa de estudios de Música.

Tabla 1.

Reconoce Visualmente

\begin{tabular}{|c|c|c|c|c|c|c|c|c|}
\hline & \multicolumn{2}{|c|}{ Deficiente } & \multicolumn{2}{|c|}{ Regular } & \multicolumn{2}{|c|}{ Bueno } & \multicolumn{2}{|c|}{ Total } \\
\hline $\begin{array}{l}\text { Reconoce } \\
\text { Visualmente }\end{array}$ & $\mathrm{Fi}$ & $\%$ & $\mathrm{Fi}$ & $\%$ & $\mathrm{Fi}$ & $\%$ & $\mathrm{Fi}$ & $\%$ \\
\hline $\begin{array}{l}\text { Familias de } \\
\text { instrumentos } \\
\text { de la orquesta }\end{array}$ & 10 & $67 \%$ & 4 & $27 \%$ & 1 & $7 \%$ & 15 & $100 \%$ \\
\hline $\begin{array}{l}\text { Instrumentos } \\
\text { eléctricos }\end{array}$ & 11 & $73 \%$ & 3 & $20 \%$ & 1 & $7 \%$ & 15 & $100 \%$ \\
\hline $\begin{array}{l}\text { Instrumentos } \\
\text { y objetos } \\
\text { sonoros del } \\
\text { entorno }\end{array}$ & 10 & $67 \%$ & 5 & $33 \%$ & 0 & $0 \%$ & 15 & $100 \%$ \\
\hline
\end{tabular}

Según la Tabla 1 en relación al Pre test: reconoce visualmente, se tiene que el $67 \%$ de los alumnos tienen un nivel Deficiente, con relación al indicador: Reconoce visualmente familias de instrumentos de la orquesta, mientras que para el indicador instrumentos eléctricos e Instrumentos y objetos sonoros del entorno se tiene un resultado de un nivel deficiente en un $73 \%$ y $67 \%$ respectivamente. Un instrumento musical es un sistema para producir uno o más tonos agradables. Los instrumentos musicales son utilizados por sus intérpretes para transferir la notación simbólica de una composición musical a los sonidos correspondientes. Un instrumento musical consiste en la combinación de uno o más sistemas de resonancia capaces de producir uno o más tonos y medios para excitar aquellos sistemas que están bajo el control de sus jugadores. Los instrumentos musicales pueden clasificarse según las categorías enumeradas en la tabla y cómo se produce el sonido. (Eumus, 2020)

Tabla 2.

Discrimina Auditivamente

\begin{tabular}{|c|c|c|c|c|c|c|c|}
\hline & De & iciente & $\mathbf{R e}$ & gular & Bueno & Tot & \\
\hline $\begin{array}{l}\text { Discrimina } \\
\text { auditivamente }\end{array}$ & $\mathrm{Fi}$ & $\%$ & $\mathrm{Fi}$ & $\%$ & $\mathrm{Fi} \%$ & $\mathrm{Fi}$ & $\%$ \\
\hline $\begin{array}{l}\text { Familias de } \\
\text { instrumentos } \\
\text { de la orquesta }\end{array}$ & 10 & $67 \%$ & 5 & $33 \%$ & $0 \%$ & 15 & $100 \%$ \\
\hline $\begin{array}{l}\text { Instrumentos } \\
\text { eléctricos }\end{array}$ & 12 & $80 \%$ & 3 & $20 \%$ & $0 \%$ & 15 & $100 \%$ \\
\hline $\begin{array}{l}\text { Instrumentos } \\
\text { y objetos } \\
\text { sonoros del } \\
\text { entorno }\end{array}$ & 13 & $87 \%$ & 2 & $13 \%$ & $0 \%$ & 15 & $100 \%$ \\
\hline
\end{tabular}


Según la Tabla 2 se observa los resultados del pre test de la dimensión: discrimina Auditivamente el nivel de los estudiantes es deficiente.

Analizar el nivel de la discriminación auditiva es importante porque ocurre cuando escuchamos y pensamos en lo que escuchamos. Es superior a la percepción porque debemos tener una razón para distinguir entre un tono y otro.

Tabla 3.

Interpretación Rítmica.

\begin{tabular}{|c|c|c|c|c|}
\hline & Deficient & Regular & Bueno & Total \\
\hline $\begin{array}{l}\text { Interpretación } \\
\text { Rítmica }\end{array}$ & $\mathrm{Fi} \quad \%$ & $\mathrm{Fi} \%$ & $\mathrm{Fi} \%$ & $\mathrm{Fi} \%$ \\
\hline $\begin{array}{l}\text { Manejo del software } \\
\text { Sibelius y Finale }\end{array}$ & $1280 \%$ & $320 \%$ & $0 \quad 0 \%$ & $15100 \%$ \\
\hline $\begin{array}{l}\text { Introduce notas } \\
\text { musicales en el } \\
\text { software }\end{array}$ & $1280 \%$ & $320 \%$ & $0 \quad 0 \%$ & $15100 \%$ \\
\hline $\begin{array}{l}\text { Realiza } \\
\text { interpretaciones } \\
\text { rítmicas: pulso, } \\
\text { acento, tiempo }\end{array}$ & $1280 \%$ & $320 \%$ & $0 \quad 0 \%$ & $15100 \%$ \\
\hline
\end{tabular}

Según la Tabla 3, los resultados del pre test para la dimensión: interpretación rítmica el $80 \%$ tiene un nivel deficiente.

Las habilidades relacionadas con los problemas de ritmo y métrica son fundamentales para las competencias musicales y para la aplicación adecuada del lenguaje musical en todas las áreas de las actividades musicales especializadas y van más allá del aprendizaje enfocado. La aplicación efectiva de las habilidades rítmicas es de particular importancia para la comprensión teórica y analítica, para la lectura y su aplicación para la interpretación musical. (Rivas, 2020)

Tabla 4.

Introduce notas en la partitura.

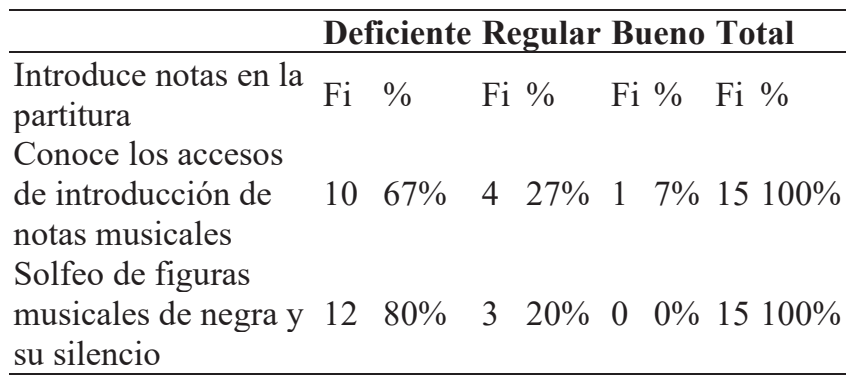

Según la Tabla 4, la dimensión: introduce notas en la partitura en el pre test se tiene que el $67 \%$ de los alumnos tienen un nivel deficiente con relación al indicador conoce los accesos de introducción de notas musicales, mientras que para el indicador solfeo de figuras musicales de negra y su silencio se tiene un resultado de un nivel deficiente en un $80 \%$. Los resultados muestran relación con los resultados de la investigación de Lituma (2015), quien hace referencia a que el manejo de programas editores de partituras ayuda al aprendizaje musical de los estudiantes porque están pensadas para permitir editar, modificar e imprimir en lenguaje musical desde una simple melodía hasta una partitura orquestal con un resultado gráfico impecable.

Tabla 5.

Teoría Musical.

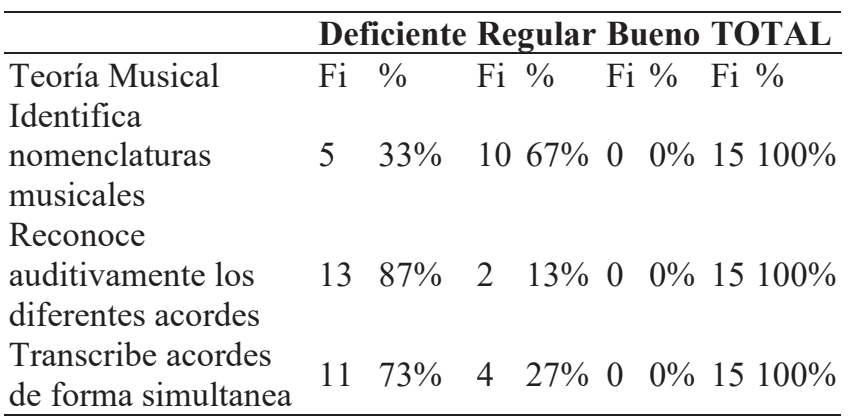

Según la Tabla 5 de resultados del pre test para la dimensión: interpretación rítmica un promedio de más del 50\% tiene un nivel deficiente.

Aprender música sin comprender su teoría es como querer estudiar literatura sin saber leer y escribir. Si bien ciertas habilidades musicales se pueden desarrollar sin teoría, el proceso es más fácil y completo cuando se comprenden al menos sus conceptos básicos. Dejar de lado la teoría musical significa ignorar los conocimientos adquiridos a lo largo de cientos de años que sin duda enriquece, perfecciona y permite entender la música para poder tocarla con mayor destreza.

La teoría es el legado que ha simplificado el entendimiento de la música. Cada vez que se aportan nuevas ideas, este conocimiento se puede reutilizar gracias a la teoría. Al entender lo que sucede en cada estilo, podemos replicarlos, transformarlos y mezclarlos adecuadamente. En música, el término interpretación se utiliza para referirse a una interpretación musical en un instrumento. Un verdadero músico interpreta música cuando la toca porque no solo sabe cómo reproducir los sonidos correctos en el momento adecuado, sino que también comprende a nivel teórico lo que está sucediendo en cada momento musical. (Colomer, 2017). 
Tabla 6.

Dictado Melódico.

\begin{tabular}{lllllllll}
\hline & \multicolumn{6}{c}{ Deficiente Regular Bueno Total } \\
\hline $\begin{array}{l}\text { Dictado melódico } \\
\text { Identifica intervalos }\end{array}$ & Fi & $\%$ & Fi $\%$ & Fi $\%$ & Fi $\%$ \\
$\begin{array}{l}\text { musicales y grados } \\
\text { tonales }\end{array}$ & 11 & $73 \%$ & 4 & $27 \%$ & 0 & $0 \%$ & 15 & $100 \%$ \\
$\begin{array}{l}\text { Transcribe entona e } \\
\text { identifica los }\end{array}$ & 11 & $73 \%$ & 3 & $20 \%$ & 1 & $7 \%$ & 15 & $100 \%$ \\
intervalos musicales & & & & & & & & \\
\hline
\end{tabular}

Según la Tabla 6, respecto a la dimensión: dictado melódico en el pre test, se tiene que el $73 \%$ de los alumnos tienen un nivel deficiente con relación al indicador identifica intervalos musicales, mientras que para el indicador transcribe entona e identifica los intervalos musicales.

El estudio del dictado musical debe estar bien enfocado desde el principio, de lo contrario puede resultar muy estresante para el alumno y llegar a convencerse de que no sirve cuando en realidad ha sido víctima de un mal planteamiento, una falta de comprensión absoluta de lo que es, tuvo que hacer y, en última instancia un malentendido para traducir sus conocimientos rítmicos, melódicos y teóricos en una hoja de papel con solo escuchar música (Dreams, 2017).

Tabla 7.

Reconoce Visualmente.

\begin{tabular}{|c|c|c|c|c|c|}
\hline & De & iciente & Regular & Bueno & TOTAL \\
\hline $\begin{array}{l}\text { Reconoce } \\
\text { Visualmente }\end{array}$ & $\mathrm{Fi}$ & $\%$ & $\mathrm{Fi} \%$ & $\mathrm{Fi} \%$ & $\mathrm{Fi} \%$ \\
\hline $\begin{array}{l}\text { Familias de } \\
\text { instrumentos de la } \\
\text { orquesta }\end{array}$ & 1 & $7 \%$ & $27 \%$ & $1067 \%$ & $015100 \%$ \\
\hline $\begin{array}{l}\text { Instrumentos } \\
\text { eléctricos }\end{array}$ & 0 & $0 \%$ & $320 \%$ & $1280 \%$ & \% 15 100\% \\
\hline $\begin{array}{l}\text { Instrumentos y } \\
\text { objetos sonoros del } \\
\text { entorno }\end{array}$ & 0 & $0 \%$ & $20 \%$ & $1280 \%$ & $015100 \%$ \\
\hline
\end{tabular}

\section{Resultados de aplicación del Post Test}

Según la Tabla 7 podemos observar que después de la aplicación de los softwares Sibelius y Finale los niveles de las capacidades musicales de los alumnos han mejorado significativamente ya que para la dimensión: reconoce visualmente se tiene un $67 \%$ con nivel bueno para el indicador familias de instrumentos de la orquesta y $80 \%$ en los indicadores Instrumentos eléctricos e Instrumentos y objetos sonoros del entorno respectivamente.
Cano (2017), manifesto que es la capacidad de conceptualizar la terminología musical, identificando, comprendiendo, relacionando y transfiriendo todos aquellos conocimientos declarativos que constituyen los materiales que utiliza el discurso musical.

Tabla 8.

Discrimina Auditivamente.

\begin{tabular}{|c|c|c|c|c|}
\hline & Deficiente & Regular & Bueno & TOTAL \\
\hline $\begin{array}{l}\text { Discrimina } \\
\text { auditivamente }\end{array}$ & $\mathrm{Fi} \quad \%$ & $\mathrm{Fi} \%$ & $\mathrm{Fi} \%$ & $\mathrm{Fi} \%$ \\
\hline $\begin{array}{l}\text { Familias de } \\
\text { instrumentos de la } \\
\text { orquesta }\end{array}$ & $7 \%$ & $20 \%$ & $1173 \%$ & $15100 \%$ \\
\hline $\begin{array}{l}\text { Instrumentos } \\
\text { eléctricos }\end{array}$ & $0 \%$ & $213 \%$ & $1387 \%$ & $015100 \%$ \\
\hline $\begin{array}{l}\text { Instrumentos y } \\
\text { objetos sonoros del } \\
\text { entorno }\end{array}$ & $0 \%$ & $4 \quad 27 \%$ & $1173 \%$ & $15100 \%$ \\
\hline
\end{tabular}

Según la Tabla 8 se observa que después de la aplicación del software Sibelius y Finale la dimensión discrimina auditivamente mejoró en sus tres indicadores ya que en el post test, los alumnos presentan un nivel bueno con porcentajes de $73 \%, 87 \%$ y $73 \%$. La discriminación auditiva debe desarrollarse paulatinamente, partiendo de sólidos juegos de localización, su práctica debe preceder al canto, y debe ser imprescindible en los primeros años de vida, cuando los niños por su gran curiosidad, tienen una mayor tendencia a aprender cosas nuevas, una de las principales características de los estudiantes (Danvila, 2010).

Tabla 9.

Interpretación Rítmica.

\begin{tabular}{|c|c|c|c|c|}
\hline & Deficiente & Regular & Bueno & Total \\
\hline $\begin{array}{l}\text { Interpretación } \\
\text { Rítmica }\end{array}$ & $\mathrm{Fi} \quad \%$ & $\mathrm{Fi} \%$ & $\mathrm{Fi} \%$ & Fi \% \\
\hline $\begin{array}{l}\text { Manejo del software } \\
\text { Sibelius y Finale }\end{array}$ & $0 \%$ & $853 \%$ & $7 \quad 47 \%$ & $15100^{\circ}$ \\
\hline $\begin{array}{l}\text { Introduce notas } \\
\text { musicales en el } \\
\text { software }\end{array}$ & $0 \%$ & $33 \%$ & $1067 \%$ & $15100 \%$ \\
\hline $\begin{array}{l}\text { Realiza } \\
\text { interpretaciones } \\
\text { rítmicas: pulso, } \\
\text { acento, tiempo }\end{array}$ & $0 \%$ & $320 \%$ & $1280 \%$ & $15100^{\circ}$ \\
\hline
\end{tabular}

Según la Tabla 9 en relación al indicador introduce notas musicales en el software se tiene un resultado de $67 \%$ con nivel bueno y un $33 \%$ con nivel regular finalmente para el indicador realiza interpretaciones rítmicas: pulso, acento, tiempo al 
igual que los demás indicadores se mejoró el nivel de deficiente a bueno en un $80 \%$.

La interpretación y el análisis musical son dos ramas de gran importancia en la concepción, creación y desarrollo de la música. Cada uno de ellos aporta propiedades fundamentales que contribuyen a la configuración de su vasto universo (Ainhoa, 2007).

Tabla 10.

Introduce notas en la partitura.

\begin{tabular}{|c|c|c|c|c|c|}
\hline & \multicolumn{4}{|c|}{ Deficiente Regular Bueno } & Total \\
\hline $\begin{array}{l}\text { Introduce notas en } \\
\text { la partitura }\end{array}$ & $\mathrm{Fi}$ & $\%$ & $\mathrm{Fi} \%$ & $\mathrm{Fi} \%$ & $\mathrm{Fi} \%$ \\
\hline $\begin{array}{l}\text { Conoce los accesos } \\
\text { de introducción de } \\
\text { notas musicales }\end{array}$ & 0 & $0 \%$ & $20 \%$ & & $15100 \%$ \\
\hline $\begin{array}{l}\text { Solfeo de figuras } \\
\text { musicales de negra } \\
\text { y su silencio }\end{array}$ & 0 & $0 \%$ & $533 \%$ & $1067 \%$ & $15100 \%$ \\
\hline
\end{tabular}

Según la Tabla 10, la dimensión introduce notas en las partituras, tiene dos indicadores que han sido evaluados tanto en el pre test como el post test, de los cuales se observó que en el indicador conoce los accesos de introducción de notas musicales lográndose el objetivo por que los resultados mejoraron en un $80 \%$ a nivel bueno, mientras que para el indicador solfeo de figuras musicales de negra y su silencio observamos que aún hay un $33 \%$ de alumnos con nivel regular y un $67 \%$ con nivel bueno. Existiendo relación con los resultados de Chan, Jones, Scanlon y Joiner (2006), que el uso de herramientas para el aprendizaje, facilitan el proceso de aprendizaje.

Tabla 11.

Teoría Musical.

\begin{tabular}{|c|c|c|c|c|}
\hline & Deficiente & Regular & r Bueno & Total \\
\hline $\begin{array}{l}\text { Teoría Musical } \\
\text { Identifica }\end{array}$ & $\mathrm{Fi} \quad \%$ & $\mathrm{Fi} \quad \%$ & $\mathrm{Fi} \%$ & Fi \% \\
\hline $\begin{array}{l}\text { nomenclaturas } \\
\text { musicales }\end{array}$ & $7 \%$ & $27 \%$ & \% $1067 \%$ & $15100 \%$ \\
\hline $\begin{array}{l}\text { Reconoce } \\
\text { auditivamente los } \\
\text { diferentes acordes }\end{array}$ & $7 \%$ & $13 \%$ & o $1280 \%$ & $15100 \%$ \\
\hline $\begin{array}{l}\text { Transcribe acordes } \\
\text { de forma } \\
\text { simultanea }\end{array}$ & $7 \%$ & $7 \%$ & $1387 \%$ & $15100 \%$ \\
\hline
\end{tabular}

Según la Tabla 11, los resultados de la dimensión: teoría musical muestran que al igual que las otras dimensiones se tiene óptimos resultados, se puede observar que el $67 \%$ de los alumnos presentan niveles buenos en el indicador identifica nomenclaturas musicales así mismo, el 80\% reconoce auditivamente los diferentes acordes y el $87 \%$ del total de los alumnos transcribe acordes de forma simultánea de manera adecuada.

La teoría permite incrementar los recursos musicales a la hora de componer. Hay cientos de métodos conocidos para crear música. Aprender estos métodos solo puede enriquecer su vocabulario $\mathrm{y}$ herramientas. La composición es un proceso creativo que se nutre de la propia experiencia y la de los demás (López, 2017)

Tabla 12.

Dictado Melódico.

\begin{tabular}{|c|c|c|c|c|}
\hline & Deficiente & Regular & Bueno & Total \\
\hline Dictado Melódico & Fi \% & $\mathrm{Fi} \%$ & Fi \% & $\mathrm{Fi} \%$ \\
\hline $\begin{array}{l}\text { Identifica intervalos } \\
\text { musicales }\end{array}$ & $0 \quad 0 \%$ & $320 \%$ & $1280 \%$ & $15100 \%$ \\
\hline $\begin{array}{l}\text { Transcribe, entona } \\
\text { e identifica los } \\
\text { intervalos } \\
\text { musicales }\end{array}$ & $0 \quad 0 \%$ & $320 \%$ & $1280 \%$ & $15100 \%$ \\
\hline
\end{tabular}

Según la Tabla 12, la investigación dio como resultado para la dimensión dictado melódico que la aplicación del software Sibelius y Finale que el $80 \%$ tiene un nivel bueno e identifica intervalos musicales como el indicador transcribe, entona e identifica así mismo el 20\% tiene un nivel regular.

El dictado melódico permite escribir una melodía con sonidos y ritmos. Estos dictados ayudan al estudiante a correlacionar lo que está escuchando y lo que está leyendo en la partitura. Transferir la audición por escrito, es decir asociar los signos de la escritura con lo que se percibe acústicamente (Pacheco, 2010).

\section{Discusión}

El resultado de la investigación está ampliamente ligado con la teoría propuesta por Herrera (2016), ya que la integración de la tecnología en la educación musical es un hecho y que los estudiantes poseen más herramientas nuevas y son más receptivos al estudio al convertirse ellos mismos en participantes activos. El proceso de aprendizaje es un desglose básico, tanto por el uso y manejo de softwares, como por el Internet de que puede considerarse como referencias obligatorias para los futuros profesores con respecto a la tecnología musical 
y sus oportunidades de enseñanza (Rodríguez, 2012). En la actualidad es necesario automatizar la composición musical mediante el apoyo de programas y/o softwares que hagan la tarea del músico más fácil.

Velazco, et al. (2020) El nivel de retención en la memoria musical de secuencias entonadas, según la aplicación del software LenMus, es significativo en un nivel de logro esperado, ya que los estudiantes mediante este software logran solfear con una entonación adecuada, reconocer los intervalos de segunda y tercera mayor y menor cuarta justa, en comparación de estudiantes que desarrollan el curso de lenguaje musical sin uso de ningún software.

Consideramos que los Softwares Sibelius y Finale son una herramienta muy importante al momento de realizar la edición y reproducción de partituras, y todo lo relacionado con la caligrafía musical. Además, estos programas facilitan la creación de composiciones audiovisuales, y lo más importante es que se puede usar de una manera rápida y fácil el manejo de notaciones y símbolos. A su vez Siamancas (2017), los software permitieron que los docentes innoven sus formas de enseñanzas de la materia de música a través del uso adecuado de los softwares musicales. Que las clases didácticas utilizando los softwares musicales favorecieron la participación y el aprendizaje de los estudiantes que intervinieron en este proceso de capacitación docente. Así mismo Buenaño (2017) expresa que las tecnologías de la información y la comunicación contribuyen a lograr una enseñanza y aprendizaje de calidad, impulsan efectivamente el desarrollo profesional de los docentes. Lenmus, Solfege y Musescore se integran satisfactoriamente y se adaptan la Metodología propuesta e influyen significativamente en el rendimiento de los estudiantes.

\section{Conclusiones}

Se concluye que la aplicación de los softwares musicales (sibelius y finale) influye positivamente en aprendizaje de los estudiantes de Música en la Universidad Nacional del Altiplano Puno logrando un nivel Muy Bueno, ya que se observó una mejora en capacidad de realizar arreglos musicales mediante los recursos informáticos, además la motivación de los estudiantes aumento considerablemente ya que les resulta más sencillo desenvolverse en el proceso de enseñanzaaprendizaje.

El nivel de las capacidades de orquestación en el pre test es de un nivel deficiente, para el post test, con la aplicación del software musical (sibelius y finale) el nivel que se logró es de Bueno, ya que los estudiantes comenzaron a comprender y desarrollar las estructuras de los conjuntos instrumentales.

Se concluye de que las capacidades en la composición mejoraron después de la aplicación de los Softwares Sibelius y Finale como herramientas vinculantes, dando como resultado en el post test mejoras en su nivel de deficiente a bueno en un $80 \%$ según muestran las tablas estadísticas como docente se evidenciaron que hubo una mejora y expansión de las utilidades de los editores de utensilios, que en este caso fueron representadas por Sibelius brindando al compositor obviamente con el conocimiento previo del dominio de estas utilidades oportunidades para la escritura musical de sus creaciones.

\section{Agradecimiento}

A la Universidad Nacional del Altiplano y los docentes por brindar educación de calidad permitiendo ser profesionales de calidad para la sociedad.

\section{Conflictos de interés}

El grupo de investigadores no tiene ningún conflicto de intereses.

\section{Referencias bibliográficas}

Agar, M. (2003). Hacia un lenguaje etnográfico. En el surgimiento de una antropología posmoderna. Edición a cargo de Carlos Reynoso. Barcelona.

Ainhoa, K. (2007). Creacion musical e ideologias. Universidad de Barcelona. Obtenido de https://www.tesisenred.net/bitstream/ handle/10803/5197/akc1de1.pdf? sequence=1

Anders, T., \& Inden, B. (2019). Aprendizaje automático de reglas de composición simbólica con programación genética: tratamiento de disonancia en Palestrina. PeerJ Ciencias de la computación, 2019(12), 1-19. doi: $10.7717 /$ peerj-cs. 244 
Ariza, C., \& Cuthbert, M. (1 de junio de 2010). Modelado de ritmos, acentos, vigas y firmas de tiempo jerárquicamente con objetos de música de 21 metros. Conferencia Internacional de Música por Computadora, ICMC 2010. 11, págs. 216-223. Nueva York y Stony Brook: Asociación Internacional de Música por Computadora.

Bannerman, J., \& O'Leary, E. (2020). Nativos digitales desconectados: supuestos desafiantes de las habilidades tecnológicas de los educadores musicales en formación. Journal of Music Teacher Education, 3(26). doi: 10.1177/1057083720951462

Barry, N. (octubre de 1992). Los efectos de las estrategias de práctica, las diferencias individuales en el estilo cognitivo y el género sobre la precisión técnica y la musicalidad de la interpretación instrumental del estudiante. Scopus, 20(2), 112-123. Doi: $10.1177 / 0305735692202002$

Bays, G., \& Zhu, Y. (18 de agosto de 2015). ScoreSVG: un editor de música basado en la web para crear partituras en SVG. 4ta Conferencia anual sobre gráficos vectoriales escalables, SVG Open 2005, (pág. 26). Países Bajos.

Belinche, M. E. (2006). Apuntes sobre Apreciación Musical. La plata: EDULP.

Bermúdez, e. (1984). Música tradicional y popular colombiana. Fascículos 10 y 11. Editorial Printer Colombiana Ltda. Procultura. Bogotá.

Best, F. (1988). Sobre el origen de la palabra "pedagogía". Perspectivas Vol. 18 F 2, p. 163172.

Borbonet, I. G. (1996). Historia de la Música. R B A Editores. Barcelona.

Buenaño, L. E. (2017). Análisis de herramientas de software libre orientadas al aprendizaje del lenguaje musical para mejorar el rendimiento académico en los estudiantes del primer año de bachillerato de la Unidad Educativa Juan de Velasco.

Calvi, J. C. (2006). La industria de la música, las nuevas tecnologías digitales e Internet. Algunas transformaciones y salto en la concentración, Zer. Revista de Estudios de Comunicación, vol. 11, $\mathrm{n}^{\mathrm{o}}$, pp. 122-137.

Campos R. y Restrepo M. (2002). La docencia como práctica: el concepto, un estilo, un modelo. Bogotá.

Canales, R. (2006). Identificación de factores que contribuyen al desarrollo de actividades de enseñanza aprendizaje con apoyo de las TIC que resulten eficientes y eficaces. Análisis de su presencia en tres centros docentes (Tesis doctoral). Universidad Autónoma de Barcelona, Barcelona.

Custodio, N. y Cano, C. M. (2017). Efectos de la música sobre las funciones Cognitivas. Revista Neuropsiquiatr 80 (1)., Lima. Obtenido de http://www.scielo.org.pe/pdf/ $\mathrm{rnp} / \mathrm{v} 80 \mathrm{n} 1 / \mathrm{a} 08 \mathrm{v} 80 \mathrm{n} 1$

Chan, L., Jones, A., Scanlon, E., \& Joiner, R. (Mayo de 2006). El uso de las TIC para apoyar el desarrollo de habilidades musicales prácticas mediante la adquisición de habilidades con el teclado: un estudio en el aula. Computadoras y educación, 46(4), 391-406. doi: 10.1016/j. compedu.2004.08.007

Chen, J. (2012). Un estudio piloto que mapea las estrategias de composición de los estudiantes: implicaciones para la enseñanza de la composición asistida por computadora. Estudios de investigación en educación musical, 34(2), 157-171. doi: 10.1177/1321103X12465515

Colomer, J. (17 de Febrero de 2017). ¿Por qué deberíamos aprender Teoría Musical? 12. Obtenido de http://www.javicolomer.com/ por-que-deberiamos-aprender-teoria-musical/

Dammers, R. (setiembre de 2012). Clases de música basadas en tecnología en escuelas secundarias de Estados Unidos. Boletín del Consejo de Investigación en Educación Musical, 9(194), 73-90. doi: 10.5406/ bulcouresmusedu.194.0073

Danvila, V. I. (2010). Las nuevas tecnologías como herramientas que facilitan la educacion formativa. Tesis. Obtenido de http://www. seeci.net/cuiciid2013/PDFs/UNIDO $\% 20$ MESA $\% 202 \% 20$ DOCENCIA.pdf

Dreams, T. A. (2017). El dictado en el lenguaje musical. Obtenido de https://toysanddreams. com/el-dictado-en-el-lenguaje-musical/

Eumus. (2003). Curso de Acústica en bachillerato. Obtenido de http://www.ehu.eus/acustica/ bachillerato/somues/somues.html

Garcia, R., \& Pelayo, G. (1995). Pequeno Larousse Ilustrado. p.709.

González, L. (2009). La música como un tipo de Lenguaje. Recuperado el 1 de octubre de 2009. Foro de Música en clave. obtenido de http:// www.musicaenclave.com/forumshowthread. php.

Haning, M. (2015). ¿Están preparados para enseñar con tecnología? Una investigación de la instrucción tecnológica en los programas de 
formación de profesores de música. Journal of Music Teacher Education, 25(3), 78-90. doi: 10.1177/1057083715577696

Hernández, S., Fernández-Collado, C., \& Baptista, P. (2006). Metodología de la investigación (4 $4^{a}$ ed.). D. F. McGraw Hill Interamericana. México.: Editores, S. A.

Herrera, J. P. (2016). Software musical y sus posibilidades didácticas: una panorámica para los estudiantes del Máster de Secundaria en la especialidad de Música. Scielo Analytics, 17.

Hormigos, J. \&. (2004). La construcción de la identidad juvenil a través de la música. Revista Española de Psicología, (4), pp. 259-270.

Huovinen, E., \& Rautanen, H. (1 de abril de 2020). Posibilidades de interacción en instrumentos tradicionales y tabletas: un estudio de la creatividad de los grupos musicales de niños. Estudios de investigación en educación musical, 42(1), 94-112. doi: $10.1177 / 1321103 X 18809510$

Jesús Tejada, M. P.-G. (2016). “Diseño y evaluación de un programa informático para la educación musical de maestros no especialistas el caso de Emolab". Revista Electrónica Complutense de Investigación en Educación Musical, 13, 28.

Julia, J., Iswara, D. D., \& Supriyadi, T. (16 de Diciembre de 2019). La utilización de la aplicación Scratch en la creación de controladores de música para introducir instrumentos musicales tradicionales. Journal Of. Psysics: conferences series, 1402(7), 4046.

Julia, J., Iswara, P., \& Supriyadi, T. (18 de Abril de 2018). Creación de canciones mediante el uso de notación musical por computadora. En A. A. Nandiyanto ABD (Ed.), 3ra Conferencia Anual de Ingeniería y Ciencias Aplicadas. 434. Indonesia: Institute of Physics Publishing. doi: 10.1088/1757-899X /434/1/012055

Julia, J., Supriyadi, T., \& Iswara, P. (5 de Mayo de 2019). El desarrollo de materiales didácticos de composición angklung utilizando notación musical Softwarecon integración de tecnología de estudio virtual. Journal of Physics: Serie de conferencias, 1157(4). doi: 10.1088/1742-6596/ 1157/4/042005

Lituma, Q. J. (2015). "El manejo de programas editores de partituras en el aprendizaje musical de los estudiantes del 6to semestre nivel técnico del conservatorio de música "salvador Bustamante celi". período febrerojulio 2013". Loja.

López, D. R. (2017). Estrategias de enseñanza creativa: investigaciones sobre la creatividad en el aula. Universidad de La Salle. Obtenido de http://biblioteca.clacso.edu.ar/Colombia/ fce-unisalle/20180225093550/estrategiasen. pdf

Naughtin, M. (2017). Preparación de música digital en la biblioteca orquestal moderna. ¿El fin de la sabiduría?: El futuro de las bibliotecas en la era digital, 87-103. doi: 10.1016/B978-008-100142-4.00009-9

Pacheco, B. D. (2010). La iniciación del estudiante de música. Universidad de Cuenca, Cuenca. Obtenido de http://dspace.ucuenca.edu.ec/ bitstream/123456789/3227/1/tdmus1.pdf

Powell, B. (1 de diciembre de 2019). La integración de la tecnología musical en los conjuntos de música popular: perspectivas de los profesores de banda modernos. Revista de música, tecnología y educación, 12(3), 297310. doi: 10.1386/jmte_00012_1

Psenicka, D. (11 de noviembre de 2016). FOMUS, una notación musical softwarepaquete para compositores de música por computadora. Conferencia Internacional de Música por Computadora, ICMC 2006. 6, págs. 75- 78. Esatdos Unidos: Asociación Internacional de Música por Computadora.

Rivas, C. N. (16 de Enero de 2020). Aprendizaje por competencias en el área de lenguaje musical para estudiantes de los colegios de artes en Ecuador. Scielo, 16(1), 15.

Rodríguez, M. (9 de Octubre de 2012). Xalan Generación algorítmica de partituras musicales expresivas basadas en análisis de señales y transformaciones gráficas. $8^{a}$ Conferencia AAAI sobre Inteligencia Artificial y Entretenimiento Digital Interactivo. 12, págs. 83- 85. Satnford: Departamento de música.

Rolando, P., \& Alvarado, A. (2013). La Musica y $\mathrm{Su}$ Rol En El Desarrollo Humano.

Siamancas, R. N. (2017). Actualización de software musical como innovación pedagógica para la enseñanza de música en los estudiantes de 5to, 6to y 7 mo de la Escuela Fiscal Mixta "24 de mayo" de la Parroquia El Valle Cantón Loja.

Smith, K. (2009). El efecto de la instrucción asistida por computadora y la independencia 
del campo en el desarrollo de las habilidades de lectura rítmica a primera vista de los estudiantes instrumentales de la escuela secundaria. Revista Internacional de Educación Musical, 27(1), 59-68.

Tejada, J. (2009). Escuchar la notación musical a través del software de partituras musicales: efectos en la lectura y escritura musical de los estudiantes. Revista internacional de aprendizaje, 16(6), 17-32. doi: 10.18848/14479494/CGP/v16i06/46351

Tejada, J., \& Pérez-Gil, M. (2016). DISEÑO Y EVALUACION DE UN PROGRAMA INFORMÁTICO PARA LA EDUCACIÓN MUSICAL DE MAESTROS NO ESPECIALISTAS. EL CASO DE EMOLAB. Revista Electrónica Complutense de Investigación en Educación Musical, 22-49. doi: 10.5209/RECIEM.52072

Tsang, H., \& McCracken, J. (agosto de 2004). Aprender términos musicales en contexto: el papel de las tecnologías de aprendizaje multimedia enriquecidas. Actas de la Séptima Conferencia Internacional IASTED sobre Computadoras y Tecnología Avanzada en Educación, 28, págs. 27-31. Kauai, HI; Estados Unidos.

Urgencias, M. (4 de octubre de 2016). Sistemas de programación de restricciones para modelar teorías musicales y composición.
Universidad de Plymouth, 43(30), 30. DOI: 10.1145/1978802.1978809

Velazco, B., Calsina, W., Valdivia, R., \& Ruelas, D. (2020). Métodos de la educación musical para el desarrollo de la memoria musical de los estudiantes música.comunic@ción, 2839. Doi:10.33595/2226-1478.11.1.431

Wise, S., Greenwood, J., \& Davis, N. (2 de julio de 2019). Uso de la tecnología digital por parte de los profesores en la educación musical secundaria: ilustraciones de aulas cambiantes. Revista británica de educación musical, 28(2), 117-134. doi: 10.1017/S0265051711000039

Wong, S., Bajo, A., \& Kang, S. L. (1 de julio de 2020). Aprender los estilos de los compositores musicales: ¿Bloquear o intercalar? Revista de investigación en educación musical, 68(2), 156-174. doi: 10.1177/0022429420908312

Yun, Y., \& Cha, S.-H. (2013). Diseño de instrumentos virtuales para música por ordenador. Revista internacional de ingeniería multimedia y ubicua, 8(5), 173-178. doi: 10.14257/ijmue.2013.8.5.16

Zimmermann, S.-A. (setiembre de 2005). Notación de pentagrama modificada que fomenta la independencia musical a través de partituras accesibles y de fácil producción. Serie de congresos internacionales, 1282(92), 11131117. doi: $10.1016 /$ j.ics.2005.05.046 